PROCEEDINGS OF THE AMERICAN MATHEMATICAL SOCIETY

Volume 135, Number 5, May 2007, Pages 1575-1578

S 0002-9939(07)08721-7

Article electronically published on January 8, 2007

\title{
EXPONENTIAL GROWTH OF LIE ALGEBRAS OF FINITE GLOBAL DIMENSION
}

\author{
YVES FELIX, STEVE HALPERIN, AND JEAN-CLAUDE THOMAS
}

(Communicated by Paul Goerss)

\begin{abstract}
Let $L$ be a connected finite type graded Lie algebra. If $\operatorname{dim} L=\infty$ and gldim $L<\infty$, then $\log$ index $L=\alpha>0$. If, moreover, $\alpha<\infty$, then for some $d, \sum_{i=1}^{d-1} \operatorname{dim} L_{k+i}=e^{k \alpha_{k}}$, where $\alpha_{k} \rightarrow \log$ index $L$ as $k \rightarrow \infty$.
\end{abstract}

We work with graded vector spaces $V$ over a field $\mathbb{k}$ of characteristic $\neq 2$ and denote by $V_{(p, q)}$ the subspace $\left\{V_{i} \mid p<i<q\right\}$. The logarithmic index of any graded vector space $V$ is defined by

$$
\log \operatorname{index} V=\lim \sup _{k} \frac{\log \operatorname{dim} V_{k}}{k},
$$

and an infinite sequence $\left(q_{i}\right)$ is a quasi-geometric growth sequence for $V$ if for some fixed $n, q_{i}<q_{i+1} \leq n q_{i}$, for all $i$, and if

$$
\frac{\log \operatorname{dim} V_{q_{i}}}{q_{i}} \longrightarrow \log \text { index } V \text {. }
$$

Now consider graded Lie algebras as defined in [4]; in particular we suppose $[x,[x, x]]=0, x \in L_{\text {odd }}$, if char $\mathbb{k}=3 . L$ is connected finite type (a cft graded Lie algebra) if $L=\left\{L_{i}\right\}_{i \geq 1}$ and each $L_{i}$ is finite dimensional. The global dimension (gldim $L$ ) and depth of a cft graded Lie algebra, $L$, are defined, respectively, by

$$
\operatorname{gldim} L=\max \left\{k \mid \operatorname{Ext}_{U L}^{k}(\mathbb{k}, \mathbb{k}) \neq 0\right\}
$$

and

$$
\operatorname{depth} L=\min \left\{k \mid \operatorname{Ext}_{U L}^{k}(\mathbb{k}, U L) \neq 0\right\} .
$$

It is easy to see that depth $L \leq \mathrm{gl} \operatorname{dim} L$.

Our main result reads:

Theorem. Suppose $L$ is a cft graded Lie algebra and $\operatorname{dim} L=\infty$. If gldim $L<\infty$, then $\log$ index $L>0$. If, moreover, $\log$ index $L<\infty$, then for some $d$,

$$
\sum_{i=1}^{d-1} \operatorname{dim} L_{k+i}=e^{k \alpha_{k}} \text {, where } \alpha_{k} \rightarrow \log \text { index } L \text {, as } k \rightarrow \infty .
$$

Received by the editors June 25, 2005 and, in revised form, February 16, 2006.

2000 Mathematics Subject Classification. Primary 55P35, 55P62, 17B70.

Key words and phrases. Homotopy Lie algebra, graded Lie algebra, global dimension, exponential growth. 
Remark. Theorem 3 of [5] establishes the same conclusion when the hypothesis gldim $L<\infty$ is weakened to depth $L<\infty$, but certain additional growth conditions on $L$ are assumed.

Now suppose $X$ is a simply connected topological space with each $H_{i}(X ; \mathbb{Q})$ finite dimensional. Then the loop space homology, $H_{*}(\Omega X ; \mathbb{Q})$, is the universal enveloping algebra of a cft graded Lie algebra $L_{X}$, isomorphic to $\pi_{*}(\Omega X) \otimes \mathbb{Q}$.

Corollary. If $\operatorname{dim} L_{X}=\infty$, gldim $L_{X}<\infty$ and $\log$ index $L_{X}<\infty$, then for some $d$,

$$
\sum_{i=1}^{d-1} \operatorname{dim} \pi_{k+i}(X) \otimes \mathbb{Q}=e^{k \alpha_{k}}
$$

where $\alpha_{k} \rightarrow \log$ index $L_{X}$ as $k \rightarrow \infty$. In particular $\sum_{i=1}^{d-1} \operatorname{dim} \pi_{k+i}(X) \otimes \mathbb{Q}$ grows exponentially in $k$.

Proof of the Theorem. First we establish

Lemma 1. An infinite-dimensional cft graded Lie algebra L of finite global dimension has a quasi-geometric growth sequence.

Proof. We use the same argument as in the proof of Theorem 2 in [5]: Put $m=$ gldim $L, a=\left(\frac{1}{2(m+1)}\right)^{m+1}$ and $\alpha=\log$ index $L$.

The Cartan-Eilenberg-Serre cochain complex $\mathcal{C}^{*}(L)$ is in fact a Sullivan algebra ([3]) of the form $\bigwedge(s L)^{\#},(s L)^{\#}$ denoting the dual of the suspension of $L$ and $\wedge V$ denoting the free graded commutative algebra on $V$. The differential in $\bigwedge(s L)^{\#}$ increases the length of word gradation by 1 and so gives a second gradation $H^{(p)}$ in $H\left(\bigwedge(s L)^{\#}\right)$. As shown in [1], $\operatorname{Ext}_{U L}^{p}(\mathbb{k}, \mathbb{k}) \cong H^{(p)}$, and so our hypothesis implies $H^{(p)}=0, p>m$.

Note that for each $k, \mathcal{C}^{*}\left(L_{\geq k}\right)$ is obtained from $\mathcal{C}^{*}(L)$ by dividing by the ideal generated by elements in $(s L)^{\#}$ of degree $\leq k$. Since gldim $L_{\geq k} \leq m$ it follows that these quotient cochain complexes also satisfy $H^{(p)}=0, p>m$. The argument of [2], section 4, can therefore be applied verbatim to $\bigwedge(s L)^{\#}$ (with cat $\bigwedge X \leq m$ replaced by gldim $L \leq m$ ) to conclude that $\alpha>0$.

The same argument in the proof of Theorem 2 in 5 now shows that each $L$ has a quasi-geometric growth sequence. Let $n_{i}$ be an increasing sequence such that $\left(\operatorname{dim} L_{n_{i}}\right)^{\frac{1}{n_{i}}}$ converges to $e^{\alpha}$. By starting the sequence at some $n_{j}$ we may assume $\operatorname{dim}(L)_{n_{i}}>\frac{1}{a}$, for all $i$. Thus the formula in ([2], top of page 189) gives a sequence $n_{i}=q_{0}<q_{1}<\cdots<q_{k}=n_{i+1}$ such that $q_{j+1} \leq(m+1) q_{j}$ and such that

$$
\left(\operatorname{dim} L_{q_{j}}\right)^{\frac{1}{q_{j}+1}} \geq\left(a \operatorname{dim} L_{n_{i}}\right)^{\frac{1}{n_{i}+1}}, \quad j<k .
$$

Hence interpolating the sequences $n_{i}$ with the sequences $q_{j}$ gives a quasi-geometric growth sequence $\left(r_{j}\right)$.

We now revert to the proof of the Theorem. Since gldim $L<\infty$ we may choose a non-zero element $x \in L$ of even degree $d$. Let $N$ be the sub-Lie algebra of elements of degree $>d$ that commute with $x$. Then

$$
\operatorname{gldim} L \geq \operatorname{gldim}(\mathbb{k} x+N)=1+\operatorname{gldim} N .
$$

If $\log$ index $N=\log$ index $L=\alpha$, then certainly $\operatorname{dim} N=\infty$, and so $N$ satisfies the hypotheses of the Theorem. By induction on global dimension it satisfies the 
conclusion. In particular, if $\alpha<\infty$, then for some $d$,

$$
\sum_{j=1}^{d-1} \operatorname{dim} N_{k+j}=e^{k \beta_{k}}
$$

with $\beta_{k} \rightarrow \alpha$. Write

$$
\sum_{j=1}^{d-1} \operatorname{dim} L_{k+j}=e^{k \alpha_{k}}
$$

Then $\alpha_{k} \geq \beta_{k}$ and $\lim \sup \alpha_{k}=\alpha$ because $\alpha=\log$ index $L$. Thus $\alpha_{k} \rightarrow \alpha$, and the Theorem holds in this case.

Lemma 2. There is a sequence of finitely generated sub-Lie algebras $E(i) \subset L$ such that $\log$ index $E(i) \rightarrow \alpha$.

Proof. Otherwise for some $\varepsilon>0$ we have $\log$ index $E \leq \alpha-\varepsilon$ for every finitely generated sub-Lie algebra $E \subset L$. Construct an increasing sequence of finitely generated sub-Lie algebras, $F(i)$, and increasing sequences $\left(k_{i}\right)$ and $\left(\ell_{i}\right)$, as follows. Set $F(0)=0$, and if $F(i)$ is constructed choose $k_{i}$ and $\ell_{i}$ so that

(i) $\operatorname{dim} F(i)_{k}<e^{k(\alpha-\varepsilon / 2)}, k \geq k_{i}$,

(ii) $\operatorname{dim} L_{\ell_{i}} \geq e^{\ell_{i}(\alpha-1 / i)}$,

(iii) $\ell_{i}>(m+1) k_{i}$.

Then let $F(i+1)$ be the sub-Lie algebra generated by $F(i)$ and $L_{\ell_{i}}$.

Now let $F=\bigcup_{i} F(i)$. Since $\operatorname{dim} F_{\ell_{i}} \geq e^{\ell_{i}\left(\alpha-\frac{1}{i}\right)}$ it follows that $\log$ index $F=\alpha$. Moreover, because $F \subset L, \operatorname{gldim} F \leq m$. Thus by Lemma 1 there is an infinite sequence $q_{j}$ such that for all $j, q_{j}<q_{j+1} \leq(m+1) q_{j}$ and $\operatorname{dim} F_{q_{j}} \geq e^{q_{j}(\alpha-\varepsilon / 2)}$. In particular we may choose $i$ and $j$ so that $q_{j} \leq k_{i}<q_{j+1}$. But then $q_{j+1} \leq$ $(m+1) q_{j} \leq(m+1) k_{i}<\ell_{i}$, and it follows that $F_{q_{j+1}}=F(i)_{q_{j+1}}$. This implies that $\operatorname{dim} F_{q_{j+1}}<e^{q_{j+1}(\alpha-\varepsilon / 2)}$, a contradiction.

Finally, we complete the proof of the theorem. It remains to consider the case $\log$ index $N<\log$ index $L$. Let $E(i) \subset L$ be finitely generated sub-Lie algebras such that $\log$ index $E(i) \rightarrow \log$ index $L$. Moreover, gldim $E(i) \leq m$ and, according to Lemma 1, each $E(i)$ has a quasi-geometric growth sequence. Since $E(i)$ is finitely generated, Theorem 3 of [5] applies and states that for some $d_{i}, \frac{\log \operatorname{dim} E(i)_{\left(k, k+d_{i}\right)}}{k}$ converges to log index $E(i)$.

Fix $\varepsilon>0$ and choose $i$ so that $\log$ index $E(i) \geq \alpha-\varepsilon / 4$. Then choose $k_{0}$ so that

$$
\frac{\log \operatorname{dim} E(i)_{\left(k, k+d_{i}\right)}}{k} \geq \alpha-\varepsilon / 3, \quad k \geq k_{0} .
$$

This implies that $k_{0}$ extends to an infinite sequence $\left(k_{\ell}\right)$ such that $k_{\ell}<k_{\ell+1}<$ $k_{\ell}+d_{i}$ and such that

$$
\frac{\log \operatorname{dim} L_{k_{\ell}}}{k_{\ell}} \geq \alpha-\varepsilon / 2, \quad \ell \geq 0 .
$$

On the other hand, since $\log$ index $N<\log$ index $L$ we may assume (for $k_{0}$ sufficiently large and $\varepsilon$ sufficiently small) that

$$
\sum_{j \leq d_{i} / d} \operatorname{dim} N_{k_{\ell}+j d} \leq \frac{1}{2} \operatorname{dim} L_{k_{\ell}}, \quad \text { for all } \ell .
$$


Since $N=(\operatorname{ker} \operatorname{ad} x)_{>d}$ we have

$$
\operatorname{dim} L_{k_{\ell}+p d} \geq \operatorname{dim} L_{k_{\ell}}-\sum_{j=0}^{p-1} \operatorname{dim} N_{k_{\ell}+j d} \geq \frac{1}{2} \operatorname{dim} L_{k_{\ell}}, \quad p \leq d_{i} / d .
$$

It follows that for $p \leq d_{i} / d$ and $k_{\ell}$ sufficiently large

$$
\frac{\log \operatorname{dim} L_{k_{\ell}+p d}}{k_{\ell}+p d} \geq \frac{\log \frac{1}{2}+\log \operatorname{dim} L_{k_{\ell}}}{k_{\ell}} \cdot \frac{k_{\ell}}{k_{\ell}+p d} \geq \alpha-\varepsilon .
$$

This establishes the Theorem.

\section{REFERENCES}

[1] H. Cartan and S. Eilenberg, Homological algebra, Princeton University Press, 1956. MR0077480 (17:1040e)

[2] Y. Felix, S. Halperin and J.-C. Thomas, The homotopy Lie algebra for finite complexes, Publications Mathématiques de l'I.H.E.S. 56 (1983), 179-202. MR0686046 (85c:55010)

[3] Y. Felix, S. Halperin and J.-C. Thomas, Rational Homotopy Theory, Graduate Texts in Mathematics 205, Springer-Verlag, 2000. MR1802847 (2002d:55014)

[4] Y. Felix, S. Halperin and J.-C. Thomas, Growth and Lie brackets in the homotopy Lie algebra, Homology, Homotopy and Applications 4 (2002), 219-225. MR.1918190 (2003g:55014)

[5] Y. Felix, S. Halperin and J.-C. Thomas, An asymptotic formula for the ranks of the homotopy groups of a finite complex, preprint 2005.

Institut Mathematique, Université Catholique de Louvain, 2, Chemin du Cyclotron, 1348, Louvain-La-Neuve, Belgium

Department of Mathematics, University of Maryland, College Park, Maryland $20742-3281$

Faculté des Sciences, Université D’Angers, 49045 Bd Lavoisier, Angers, France 